\section{P-75 DEVELOPING A VOLUNTEER WORKFORCE FOR A LARGE LYMPHOEDEMA SERVICE TO SUPPORT THE CLINICAL TEAM TO CONTINUE SERVICES TO PATIENTS DURING THE COVID-19 PANDEMIC}

Laura Lowther, Angela Egdell, Kath Clark. St Oswald's Hospice, Newcastle upon Tyne, UK

10.1136/spcare-2021-Hospice.93

Background St Oswald's provides a large and specialist Lymphoedema Service which has traditionally been predominantly clinic-based at their main hospice site. Due to the impact of COVID-19, the service had to quickly pivot to provide much more domiciliary based care - to adhere to new infection control measures and respond to shielding patients' needs. To adhere to the practical nature of the care as well as safeguarding regulations, the domiciliary visits had to be carried out by two lymphoedema nurses and it became apparent that recruitment of additional volunteers would be extremely beneficial and allow more patients to benefit from care.

Aims To increase the number of volunteers recruited and inducted into their role to allow the lymphoedema service to maintain services during the pandemic.

Methods To respond to an increased need for domiciliary care, in April 2020 St Oswald's Hospice begun recruitment by advertising the volunteer role on social media. Following recruitment, volunteers were inducted and trained. During this time, staff engagement sessions were carried out and a clinical Volunteering Steering Group was set up to review and evaluate the project.

Results Over 40 new volunteers recruited, with 20-30 currently volunteering in the service. Other benefits have included: meaningful collaboration between a lymphoedema service and volunteering department, funding sourced for a volunteer coordinator for lymphoedema service to continue to build on this success, and reaching new volunteer audiences as the role engaged a much younger cohort of volunteers than traditional volunteers.

Conclusion The rapid flexing of the service supported by the Volunteering Department allowed services to be maintained, patients continued to receive assessment and treatment at home, which many prefer. In addition an unseen benefit has been volunteer enjoyment and a reporting that the experience of volunteering has been beneficial in particular in seeking university and other employment placements.

\section{P-76 TRANSFORMING A HOSPICE COUNSELLING SERVICE TO CONTINUE WORKING THROUGH THE COVID-19 PANDEMIC}

Seamus Nash, Rosie Dempsey, Tracey Addy. The Kirkwood, Huddersfield, UK

10.1136/spcare-2021-Hospice.94

It can be argued that facilitating patients and clients to embrace, manage and adapt to change can be considered as fundamental aspects to counselling. At the Kirkwood the counselling team have had to change how we deliver services and in turn how we work.

In the first instance the team were sent home to provide counselling services via telephone and video calls. The team had to adapt to this instantly. Firstly we sought advice and had training through our respective membership organisations in order to ensure we worked safely and ethically. We then had to contact every client known to us to brief them on immediate changes. Our bereavement support volunteers continued to carry out phone calls. The counsellors were then trained in AccuRx and Zoom to provide video calls. The team also attended various on-line forums for support and to keep abreast of new initiatives. The team developed two 8 week courses on Anxiety Management and Mindfulness for Emotional Well-Being, a newly bereaved counselling group and bereavement therapy group; all facilitated via Zoom.

Learnings Good planning and IT support are essential to help with technical issues and training on various platforms. Clients had work contact numbers of the facilitators which allowed for more personal support if needed when difficult issues arose. The virtual groups were extremely well received by isolated clients; and AccuRx also helped patients. We learned that patients, carers and post-bereavement clients required, at least, one face-to-face session. This enabled more accurate assessments of need and support. This meeting, according to feedback, was vital in enabling patients and clients to see a 'real' person, and thus cement trust in the therapeutic relationship.

\section{\begin{tabular}{|l|l}
\hline P-77 TRANSFORMATION OF THE COMPLEMENTARY THERAPY \\
\hline
\end{tabular} SERVICE}

Liesl Hopkins. St Richard's Hospice, Worcester, UK

\subsection{6/spcare-2021-Hospice.95}

Background With a long waiting list and staff therapists working from home, we came up with very creative ideas to continue to provide therapeutic support during the pandemic. Complementary therapies to help improve energy levels, support with breathlessness, reduce anxiety and stress, help with medication, help with relaxation, improve mood and aid sleep.

Aim(s) To continue to provide complementary therapies to patients on the Therapeutic Programme, Outpatients, Carers and Bereaved, Inpatients.

Methods We continue to provide one-to-one complementary therapies to people on our caseload including teaching carers, self-massage, Indian Head Massage, Relaxation Therapy and HEARTS Therapy. All treatments are carried out virtually under the guidance of a qualified complementary therapist. Feedback cards are completed and returned to us; reporting high quality therapy treatments have continued throughout the pandemic.

Results Pre-pandemic 2019; 133 aromatherapy massages and 270 reflexology treatments were given.

Pandemic, 2020-2021; 43 HEARTS treatments, 77 massage therapy and 87 relaxation therapy treatments were given, indicating a positive shift away from hands-on to talking therapies.

Jan. 2019 to May 2019 - 321 treatments were given; Jan. 2020 to May 2020 - 139 treatments were given; Jan. 2021 to May 2021 - 243 treatments were given.

Complementary therapies remained ever popular during lockdown.

Conclusions The complementary therapy team intend to provide a hybrid model of care in the future with virtual therapy treatments alongside face-to-face for patients, carers and bereaved. This will enable us to support a wider spectrum of people in South Worcestershire. 